\title{
THE COMMUTING BLOCK MAPS PROBLEM
}

BY

\author{
ETHAN M. COVEN, G. A. HEDLUND AND FRANK RHODES
}

\begin{abstract}
A block map is a map $f:\{0,1\}^{n} \rightarrow\{0,1\}$ for some $n>1$. A block map $f$ induces an endomorphism $f_{\infty}$ of the full 2-shift $(X, \sigma)$. We define composition of block maps so that $(f \circ g)_{\infty}=f_{\infty} \circ g_{\infty}$. The commuting block maps problem (for $f$ ) is to determine $e(f)=\{g \mid f \circ g=g \circ f\}$. We solve the commuting block maps problem for a number of classes of block maps.
\end{abstract}

Introduction. Let $(X, \sigma)$ denote the shift dynamical system over $\{0,1\}$, also called the full 2-shift. Thus $X$ is the set of bisequences of zeros and ones,

$$
X=\prod_{-\infty}^{\infty}\{0,1\}=\left\{x=\left(x_{i}\right) \mid x_{i}=0 \text { or } 1 ; i=0, \pm 1, \ldots\right\} .
$$

We assign the discrete topology to $\{0,1\}$ and the product topology to $X$, thus making $X$ homeomorphic to the Cantor discontinuum. The shift homeomorphism $\sigma: X \rightarrow X$ is defined by $[\sigma(x)]_{i}=x_{i+1}$.

An endomorphism of $(X, \sigma)$ is a continuous, shift-commuting map of $X$ to itself. Much is known about the endomorphisms of $(X, \sigma)$-see the survey article $[\mathbf{H}]$. However, little is known about their commuting properties. For example, given an endomorphism $F$, can one determine $\{G \mid F \circ G=G \circ F\}$ ?

Let $\mathbf{Z}_{2}=\{0,1\}$ denote the ring of integers modulo 2 and let $n \geqslant 1$. An $n$-block is a sequence $b_{1} \cdots b_{n}$ where $b_{i}=0$ or 1 for $1 \leqslant i \leqslant n$. An $n$-block map is a function from the set of all $n$-blocks to $\{0,1\}$. The set of all $n$-block maps will be denoted $\mathscr{F}_{n}$. A block map is a member of $\mathscr{F}=\bigcup_{n=1}^{\infty} \mathscr{F}_{n}$, i.e., an $n$-block map for some $n$.

A block map $f$ induces an endomorphism $f_{\infty}$ of $(X, \sigma)$ defined as follows. If $f$ is an $n$-block map, then

$$
\left[f_{\infty}(x)\right]_{i}=f\left(x_{i} \cdots x_{i+n-1}\right) .
$$

A theorem of Curtis, Hedlund and Lyndon [H, Theorem 3.4] asserts that every endomorphism of $(X, \sigma)$ is of the form $\sigma^{m} \circ f_{\infty}$ for some block map $f$ and some integer $m$. We will define equality of block maps so that $f=g$ if and only if $f_{\infty}=g_{\infty}$, and composition of block maps so that $(f \circ g)_{\infty}=$ $f_{\infty} \circ g_{\infty}$. This will allow us to turn questions about endomorphisms into

Received by the editors February 25, 1977.

AMS (MOS) subject classifications (1970). Primary 54H20.

(ㄷ) 1979 American Mathematical Society 0002-9947/79/0000-0154/\$07.50 
questions about block maps. Questions about block maps are often quite susceptible to solution as they can be phrased in finite combinatorial terms.

For $f \in \mathscr{F}$, let

$$
\mathcal{C}(f)=\{g \in \mathscr{F} \mid f \circ g=g \circ f\} .
$$

The commuting block maps problem (for $f$ ) is to determine $\mathcal{C}(f)$. The solution to the analogously defined "commuting endomorphisms problem" follows from the solution to the commuting block maps problem. If $F=\sigma^{m} \circ f_{\infty}$ is an endomorphism, then $\{G \mid F \circ G=G \circ F\}=\left\{\sigma^{n} \circ g \mid n \in \mathbf{Z}, g \in \mathcal{C}(f)\right\}$. In this paper, we solve the commuting block maps problem for a number of classes of block maps.

A polynomial function in $n$ variables over $\mathbf{Z}_{2}$ is a function of the form

$$
\sum a_{i_{1}} \cdots i_{n} x_{1}^{i_{1}} \cdots x_{n}^{i_{n}}
$$

where $i_{1} \cdots i_{n}$ ranges over the set of $n$-blocks. Any such function defines an $n$-block map. For example, the polynomial

$$
x_{1}+x_{2}+x_{2} x_{3}+1
$$

defines a 3-block map. It is well known [H, Theorem 19.1] that distinct polynomial functions define distinct $n$-block maps. An elementary counting argument then shows that the set $\mathscr{F}_{n}$ of all $n$-block maps is identical with the set of polynomial functions in $n$ variables over $Z_{2}$-each set has $2^{2^{n}}$ members.

Although the formal presentation of the theory in this paper makes no use of the polynomial representation of block maps, the commuting block maps problem was originally posed and solved in terms of polynomials. Indeed, the definitions and the statements of the results (although not necessarily the proofs) are often better understood in the language of polynomials. We shall sometimes provide the translation into this language.

The paper is structured as follows. $\S 1$ is devoted to preliminaries. Much of the terminology and notation introduced is either new or differs from the standard terminology and notation of $[\mathbf{H}]$. $\$ 2$ deals with an important class of block maps, those which are linear in the first variable. $\$ 3$ contains the solution to the commuting block maps problem for the class of linear block maps, those of the form $a_{0}+\sum a_{i} x_{i}$. In $\S 4$ we show that if $f\left(x_{0} \cdots x_{k}\right)=x_{0}$ $+\varphi\left(x_{1} \cdots x_{k}\right)$ where $k \geqslant 2$ and the full product $x_{1} x_{2} \cdots x_{k}$ is a summand of $\varphi\left(x_{1} \cdots x_{k}\right)$, then (modulo translations) every member of $\mathcal{C}(f)$ is the sum of an odd number of powers of $f$. In $\S \S 5$ and 6 we solve the commuting block maps problem for $f\left(x_{0} \cdots x_{k}\right)=x_{0}+\Pi_{i=1}^{k}\left(x_{i}+\delta_{i}\right)$ where $k>2$. We show that (modulo translations) $\mathcal{C}(f)$ consists of either the set of all sums of an odd number of powers of $f$, or the set of all powers of $f$, depending on the least period of the $k$-block $\delta_{1} \cdots \delta_{k}$.

It appears that a major stumbling block to a full solution to the commuting 
block maps problem is the lack of any real understanding of factorization (with respect to composition) of block maps, the only exception being the case of linear maps, where the factorization problem was completely solved by J. D. Ferguson [F]. For example, the results of $\$ \S 4-6$ cited above can hold only for maps which have no commuting factors. Yet we have no method of determining whether a given map even has any proper factors.

It should be pointed out that our methods are peculiar to the full 2-shift. They rely heavily on the algebraic structure that the set of block maps inherits from $\mathbf{Z}_{2}$. The additive structure breaks down for subshifts and the multiplicative structure becomes much more complicated for full shifts on more than two symbols.

The authors wish to thank J. D. Ferguson for pointing out that our original results of $\$ 4$ could be extended to a class of maps much larger than that considered in $\$ \S 5$ and 6.

1. Preliminaries. Under the usual definition of equality of functions (in particular, equal functions have the same domain), the correspondence between $f$ and $f_{\infty}$ is not one-to-one. For example, if $f \in \mathscr{F}_{1}$ and $g \in \mathscr{F}_{2}$ are defined by

$$
f\left(x_{1}\right)=x_{1}, \quad g\left(x_{1} x_{2}\right)=x_{1},
$$

then $f \neq g$, but both $f_{\infty}$ and $g_{\infty}$ are the identity map of $X$. Since our motivation for studying block maps comes from their connection with endomorphisms, this notion of equality is too restrictive.

We could define an equivalence relation $\sim$ on $\mathscr{F}$ by $f \sim g$ provided $f_{\infty}=g_{\infty}$, and then study $\mathscr{F} / \sim$ instead of $\mathscr{F}$. In fact, we will do this informally. We define equality in $\mathscr{F}$ as follows: if $f \in \mathscr{F}_{m}$ and $g \in \mathscr{F}_{n}$, then $f=g$ provided $f\left(b_{1} \cdots b_{m}\right)=g\left(b_{1} \cdots b_{n}\right)$ for every $N$-block $b_{1} \cdots b_{N}$ where $N=\max \{m, n\}$. It is easy to see that $f=g$ if and only if $f_{\infty}=g_{\infty}$. Furthermore, equality holds if and only if $f$ and $g$ are defined by the same polynomial.

Under this definition of equality, $\mathscr{F}_{n} \subseteq \mathscr{F}_{n+1}$ for all $n \geqslant 1$. Thus every $n$-block map may be thought of as an $(n+1)$-block map as well.

In the sequel, we shall define a number of properties of block maps and a number of operations of the set of block maps, usually by defining them on each $\mathscr{F}_{n}$ separately. We shall omit the verifications that these properties and operations are well-defined.

We define the binary operation of composition $\left({ }^{\circ}\right)$ on the set of block maps as follows. If $f \in \mathscr{F}_{m}$ and $g \in \mathscr{F}_{n}$, then $g \circ f \in \mathscr{F}_{m+n-1}$ is defined by

$$
g \circ f\left(x_{1} \cdots x_{m+n-1}\right)=g\left(y_{1} \cdots y_{n}\right)
$$

where $y_{i}=f\left(x_{i} \cdots x_{i+m-1}\right)$ for $1 \leqslant i \leqslant n$. 
For example, if $f\left(x_{1} x_{2} x_{3}\right)=x_{1}+x_{2}\left(1+x_{3}\right)$, then $g=f \circ f$ is calculated as follows.

$$
\begin{aligned}
g\left(x_{1} x_{2} x_{3} x_{4} x_{5}\right)=f\left[x_{1}+x_{2}\left(1+x_{3}\right), x_{2}+x_{3}\left(1+x_{4}\right), x_{3}+x_{4}\left(1+x_{5}\right)\right] \\
=x_{1}+x_{2}\left(1+x_{3}\right)+\left[x_{2}+x_{3}\left(1+x_{4}\right)\right]\left[1+x_{3}+x_{4}\left(1+x_{5}\right)\right] \\
=\left(x_{1}+x_{2}+x_{2} x_{3}\right)+\left(x_{2}+x_{3}+x_{3} x_{4}\right)\left(1+x_{3}+x_{4}+x_{4} x_{5}\right) \\
=\left(x_{1}+x_{2}+x_{2} x_{3}\right)+\left(x_{2}+x_{2} x_{3}+x_{2} x_{4}+x_{2} x_{4} x_{5}\right) \\
\quad+\left(x_{3}+x_{3}+x_{3} x_{4}+x_{3} x_{4} x_{5}\right)+\left(x_{3} x_{4}+x_{3} x_{4}+x_{3} x_{4}+x_{3} x_{4} x_{5}\right) \\
=x_{1}+x_{2} x_{4}+x_{2} x_{4} x_{5} .
\end{aligned}
$$

There are three particular block maps which we will find useful in the sequel. The 1-block maps $I, 0$ and 1 are defined by

$$
I\left(x_{1}\right)=x_{1}, \quad 0\left(x_{1}\right)=0, \quad 1\left(x_{1}\right)=1 .
$$

Thus $I_{\infty}$ is the identity map of $X$ and hence $I$ commutes with every block map.

We will use the power notation to denote repeated composition. Formally, $f^{0}=I$ and for $n \geqslant 0, f^{n+1}=f \circ f^{n}$. It follows easily that $f^{n+1}=f^{n} \circ f$ as well, and hence

$$
\left\{f^{n} \mid n \geqslant 0\right\} \subseteq \mathcal{C}(f) .
$$

Our (partial) solution to the commuting block maps problem relies heavily on the algebraic structure that $\mathscr{F}$ inherits from $\mathbf{Z}_{2}$. We describe this structure below.

We define the binary operations of addition $(+)$ and multiplication $(\cdot)$ on the set of block maps as follows. If $f \in \mathscr{F}_{m}$ and $g \in \mathscr{F}_{n}$, then $f+g, f \cdot g \in$ $\mathscr{F}_{N}$, where $N=\max \{m, n\}$, are defined by

$$
\begin{aligned}
(f+g)\left(b_{1} \cdots b_{N}\right) & =f\left(b_{1} \cdots b_{m}\right)+g\left(b_{1} \cdots b_{n}\right), \\
(f \cdot g)\left(b_{1} \cdots b_{N}\right) & =f\left(b_{1} \cdots b_{m}\right) \cdot g\left(b_{1} \cdots b_{n}\right) .
\end{aligned}
$$

We define addition and multiplication on $X$ and on the set of endomorphisms similarly. It follows easily that

$$
(f+g)_{\infty}=f_{\infty}+g_{\infty} \text { and }(f \cdot g)_{\infty}=f_{\infty} \cdot g_{\infty} .
$$

With operations + and $\cdot, \mathscr{F}$ is a commutative ring with identity. Since $f \cdot f=f$ for all $f$, there will be no confusion with the power notation $f^{2}=f \circ f$, which we reserve for composition.

Composition distributes over addition and multiplication as follows.

$$
(g+h) \circ f=g \circ f+h \circ f, \quad(g \cdot h) \circ f=(g \circ f) \cdot(h \circ f) .
$$

With operations + and $\circ, \mathscr{F}$ satisfies all the axioms for a ring except the right distributive law: $h \circ(f+g)$ need not equal $h \circ f+h \circ g$. For example, let $f=0, g=1$ and $h=I+1$. 
If $g \in \mathscr{F}_{n}$ commutes with $f$, then the $(n+1)$-block map $h$ defined by

$$
h\left(x_{1} \cdots x_{n+1}\right)=g\left(x_{2} \cdots x_{n+1}\right)
$$

also commutes with $f$. Therefore to determine $\mathcal{C}(f)$, we need not look at "translated" maps like $h$, whose polynomial representation does not "involve" $x_{1}$. We now introduce a formal device for effecting this reduction of the commuting block maps problem.

We define the translation operator $T$ on the set of block map as follows. If $f \in \mathscr{F}_{n}$, then $T f \in \mathscr{F}_{n+1}$ is defined by

$$
T f\left(x_{1} \cdots x_{n+1}\right)=f\left(x_{2} \cdots x_{n+1}\right) .
$$

We shall of ten abbreviate this by writing

$$
T f(0 B)=T f(1 B)=f(B) .
$$

The following properties of translation are easily derived.

(1.1) LeMma. (a) $T$ is additive, i.e., $T(f+g)=T f+T g$.

(b) $T$ is multiplicative, i.e., $T(f \cdot g)=T f \cdot T g$.

(c) $T 0=0$ and $T 1=1$.

(d) $(T f)_{\infty}=f_{\infty} \circ \sigma=\sigma \circ f_{\infty}$.

(e) $T(g \circ f)=T g \circ f=g \circ T f$.

(f) $f=g$ if and only if $T f=T g$.

The operator $T$ will be used to reduce the commuting block maps problem as indicated by the following result. (See also (1.4) and the comments following it.)

(1.2) Lemma. (a) $g \in \mathcal{C}(f)$ if and only if $T g \in \mathcal{C}(f)$.

(b) $\mathcal{C}(f)=\mathcal{C}(T f)$.

It follows from (1.2) that $\mathcal{C}(f)$ can be easily recovered from

$$
\{g \in \mathcal{C}(f) \mid g=\text { Th for some } h\} \text {. }
$$

Thus the number $\max \left\{n \mid g=T^{n-1} h\right.$ for some $\left.h\right\}$ will be of some interest to us.

Let $f$ be a block map. The left and right extents, $\lambda(f)$ and $\rho(f)$, of $f$ are defined as follows. If $f$ is constant (i.e., $f=0$ or 1 ), then $\lambda(f)=\rho(f)=0$; if $f$ is not constant, then

$$
\begin{aligned}
& \lambda(f)=\max \left\{n \mid f=T^{n-1} g \text { for some } g\right\}, \\
& \rho(f)=\min \left\{n \mid f \in \mathscr{F}_{n}\right\} .
\end{aligned}
$$

In terms of polynomials, if $f$ is an $n$-block map then $\lambda(f)$ is the smallest and $\rho(f)$ the largest subscript of a variable which appears in the polynomial representation of $f\left(x_{1} \cdots x_{n}\right)$.

We list some properties of $\lambda$ and $\rho$. 
(1.3) Lemma. Suppose $f$ and $g$ are block maps and $f$ is not constant. Then

(a) $\lambda(T f)=\lambda(f)+1$ and $\rho(T f)=\rho(f)+1$.

(b) If $f \in \mathscr{F}_{n}$, then $1 \leqslant \lambda(f) \leqslant \rho(f) \leqslant n$.

(c) $f=T^{\lambda(f)-1} h$ for some $h$ satisfying $\lambda(h)=1$.

(d) If $f \in \mathscr{F}_{n}$ with $n \geqslant 2$, then $\rho(f)=n$ if and only if $f(B 0) \neq f(B 1)$ for some $(n-1)$-block $B$.

(e) $\rho(f+g) \leqslant \max \{\rho(f), \rho(g)\}$. Equality holds if $\rho(f) \neq \rho(g)$.

We say a block map $f$ is trivial provided $\lambda(f)=\rho(f)$. It is easy to see that $f$ is trivial if and only if $f$ is constant or of the form $T^{n} I$ or $T^{n} I+1$ for some $n \geqslant 0$. For the most part, we will be concerned only with nontrivial block maps.

For each block map $f$, let

$$
\begin{aligned}
& \mathcal{C}^{\prime}(f)=\{g \in \mathcal{C}(f) \mid g \text { is constant }\}, \\
& \mathcal{C}^{\prime \prime}(f)=\{g \in \mathcal{C}(f) \mid g \text { is not constant }\}, \\
& \mathcal{C}^{*}(f)=\{g \in \mathcal{C}(f) \mid \lambda(g)=1\} .
\end{aligned}
$$

(1.4) Lemma. Let $f$ be a block map. Then $\varrho^{\prime \prime}(f)=\left\{T^{k} g \mid k \geqslant 0, g \in e^{*}(f)\right\}$.

Proof. Let $h \in \mathcal{C}^{\prime \prime}(f)$. Then $h$ is not constant, so $h=T^{\lambda(h)-1} g$ for some $g$ with $\lambda(g)=1$. Then $g \in \mathcal{C}(f)$ and so $g \in \mathcal{C}^{*}(f)$.

Let $h=T^{k} g$ for some $g \in e^{*}(f)$. Since $g$ is not constant, $\rho(g)>1$. Hence $\rho(h) \geqslant 1$ and so $h$ is not constant. But $h \in \mathcal{C}(f)$ and so $h \in \mathcal{C}^{\prime \prime}(f)$.

It follows that to solve the commuting block maps problem for $f$ it is sufficient to determine $\mathcal{C}^{\prime}(f)$ and $\mathcal{C}^{*}(f)$. The set $\mathcal{C}^{\prime}(f)$ is easily determined. If $f \in \mathscr{F}_{n}$, then $0 \in \mathcal{C}(f)$ if and only if $f\left(0^{n}\right)=0$ where $0^{n}$ is the $n$-block consisting of all zeros. The analogous result holds with 1 in place of 0 . The major portion of this paper (\$\$3-6) will be devoted to the determination of $e^{*}(f)$ for certain classes of block maps.

2. Linearity in the first variable. In this section we will be concerned with block maps of the form $f\left(x_{1} \cdots x_{n}\right)=x_{1}+\varphi\left(x_{2} \cdots x_{n}\right)$, which we will call linear in the first variable. Linearity in the first variable is important in the study of endomorphisms of $(X, \sigma)$ because it is one of the simplest known properties of a block map $f$ which implies that $f_{\infty}$ maps $X$ onto $X, f_{\infty}$ is finite-to-one, $f_{\infty}$ preserves the Bernoulli $\left(\frac{1}{2}, \frac{1}{2}\right)$-measure, as well as various other properties (see $[\mathbf{H}]$ ). The main result of this section is that if $f$ is nontrivial and linear in the first variable, then every member of $\mathcal{C}^{*}(f)$ is linear in the first variable.

Let $f$ be an $n$-block map with $n \geqslant 2$. Then we may write

$$
f\left(x_{1} \cdots x_{n}\right)=x_{1} \psi\left(x_{2} \cdots x_{n}\right)+\varphi\left(x_{2} \cdots x_{n}\right) .
$$

We may either think of $\psi$ as the "coefficient" of $x_{1}$ in $f\left(x_{1} \cdots x_{n}\right)$ or think of 
$\psi$ as the "quotient" and $\varphi$ as the "remainder" when dividing $f\left(x_{1} \cdots x_{n}\right)$ by $x_{1}$.

We introduce a formal device for picking out this quotient or coefficient. We define the quotient and remainder operators $Q$ and $R$ on the set of block maps as follows. Let $f \in \mathscr{F}_{n}$; we may assume that $n \geqslant 2$. Then $Q f, R f \in$ $\mathscr{F}_{n-1}$ are defined by

$$
Q f(B)=f(0 B)+f(1 B), \quad R f(B)=f(0 B) .
$$

The properties of $Q$ and $R$ listed below follow easily from the definitions.

(2.1) Lemma. (a) $Q$ and $R$ are additive, i.e., $Q(f+g)=Q f+Q g$ and $R(f+g)=R f+R g$.

(b) $Q T=0$, i.e., $Q T(f)=0$ for all $f$.

(c) $R T$ is the identity operator, i.e., $R T(f)=f$ for all $f$.

(2.2) LEMMA. If $f$ is not constant, then $\rho(Q f) \leqslant \rho(f)-1$.

Proof. If $\rho(f)=1$, then $f=I$ or $I+1$ and so $Q f=1$. Therefore $\rho(Q f)=$ 0. If $\rho(f)=n \geqslant 2$, then $f \in \mathscr{F}_{n}$. Thus $Q f \in \mathscr{F}_{n-1}$ and so $\rho(Q f) \leqslant n-1$.

The next result expresses equation (*) in terms of $Q$ and $R$.

(2.3) THEOREM. Let $f$ be a block map. Then

$$
f=I \cdot T Q f+T R f
$$

Furthermore, this expression is unique, i.e., if $f=I \cdot T \psi+T \varphi$, then $\psi=Q f$ and $\varphi=R f$.

Proof. Let $f \in \mathscr{F}_{n}$; we may assume that $n \geqslant 2$. Let $g=I \cdot T Q f+T R f$. To show that $g=f$, it suffices to show that for every $(n-1)$-block $B$, $g(0 B)=f(0 B)$ and $g(1 B)=f(1 B)$.

If $B$ is an $(n-1)$-block, then

$$
\begin{aligned}
& g(0 B)=0 \cdot T Q f(0 B)+T R f(0 B)=R f(B)=f(0 B), \\
& g(1 B)=1 \cdot T Q f(1 B)+T R f(1 B)=Q f(B)+R f(B)=f(1 B) .
\end{aligned}
$$

To show that the expression (**) is unique, let $f=I \cdot T \psi+T \varphi$. Then for every $(n-1)$-block $B$,

$$
f(0 B)=0 \cdot T \psi(0 B)+T \varphi(0 B)=\varphi(B),
$$

and therefore $\varphi=R f$. Similarly

$$
f(1 B)=1 \cdot T \psi(1 B)+T \varphi(1 B)=\psi(B)+\varphi(B)
$$

and hence $\psi(B)=f(0 B)+f(1 B)$, i.e., $\psi=Q f$.

As a corollary, we obtain the product rule for the quotient operator.

(2.4) Corollary. $Q(f \cdot g)=Q f \cdot Q g+Q f \cdot R g+R f \cdot Q g$. 
A block map $f$ is called linear in the first variable, written $f \in \mathfrak{L}_{1}$, provided $Q f=1$.

In terms of polynomials, an $n$-block map $f$ where $n \geqslant 2$ is linear in the first variable if and only if

$$
f\left(x_{1} \cdots x_{n}\right)=x_{1}+\varphi\left(x_{2} \cdots x_{n}\right)
$$

for some $(n-1)$-block map $\varphi$.

The following properties of $\mathscr{L}_{1}$ are easily verified.

(2.5) LemMA. (a) $I \in \mathfrak{L}_{1}$.

(b) $f \in \mathscr{L}_{1}$ if and only if $f=I+T \varphi$ for some $\varphi$.

(c) $\lambda(f)=1$ for all $f \in \mathcal{L}_{1}$.

(d) $f \in \mathfrak{L}_{1}$ is nontrivial if and only if $\rho(f) \geqslant 2$.

(e) If $f \in \mathscr{F}_{n}$ where $n \geqslant 2$, then $f \in \mathcal{L}_{1}$ if and only if $f(0 B) \neq f(1 B)$ for every $(n-1)$-block $B$.

(2.6) Lemma. If $f, g \in \mathfrak{L}_{1}$, then $g \circ f \in \mathfrak{L}_{1}$.

Proof. Write $f=I+T R f$ and $g=I+T R g$. Then $g \circ f=I+T(R f+$ $R g \circ f)$.

(2.7) COROLlaRY. If $f \in \mathscr{L}_{1}$, then $f^{n} \in \mathscr{L}_{1}$ for all $n \geqslant 0$ and hence $\left\{f^{n} \mid n \geqslant\right.$ $0\} \subseteq \mathcal{C}^{*}(f)$.

A straightforward calculation establishes the relations among the $R f^{n}$ when $f \in \mathcal{L}_{1}$.

(2.8) Lemma. If $f \in \mathcal{L}_{1}$, then for every $n \geqslant 0$,

$$
R f^{n+1}=R f+R f^{n} \circ f=R f^{n}+R f \circ f^{n} .
$$

(2.9) LeMma. Let $f \in \mathcal{L}_{1}$. Then for every $g, Q(f \circ g)=Q g$ and furthermore, for every $k \geqslant 1, Q^{k}(g \circ f)=Q^{k} g \circ f$.

Proof. Write $f=I+T R f$ and $g=I \cdot T Q g+T R g$. Then

$$
f \circ g=I \cdot T Q g+T(R g+R f \circ g)
$$

and so the first statement follows from (2.3).

We prove the second statement by induction on $k$. Since

$$
g \circ f=I \cdot[T(Q g \circ f)]+T[R f \cdot(Q g \circ f)+R g \circ f],
$$

the statement is true for $k=1$ by (2.3). Now suppose it is true for $k$. Then

$$
\begin{aligned}
Q^{k+1}(g \circ f) & =Q^{k}[Q(g \circ f)]=Q^{k}(Q g \circ f) \\
& =\left[Q^{k}(Q g)\right] \circ f=Q^{k+1} g \circ f .
\end{aligned}
$$

The next two results are due to J. D. Ferguson [F]. We include proofs because of the relative inaccessibility of $[\mathbf{F}]$. 
(2.10) LeMma [F, THeOREM 3.6]. If $f \in \mathscr{L}_{1}$ and $g$ is not constant, then

$$
\rho(g \circ f)=\rho(g)+\rho(f)-1 \text {. }
$$

Proof. Since neither $f$ nor $g$ is constant, $\rho(f) \geqslant 1$ and $\rho(g) \geqslant 1$.

If $\rho(g)=1$, then $g=I$ or $I+1$. If $g=I$, then $g \circ f=f$ and the result holds. If $g=I+1$, then $g \circ f=f+1$. Since $\rho(f+1)=\rho(f)$, the result holds in this case as well. A similar argument shows that the result is true if $\rho(f)=1$.

Thus we may assume that $\rho(f)=m, \rho(g)=n$ where $m, n \geqslant 2$ and hence that $f \in \mathscr{F}_{m}$ and $g \in \mathscr{F}_{n}$. Therefore $g \circ f \in \mathscr{F}_{m+n-1}$.

By (1.3), there exist $A=a_{1} \cdots a_{m-1}$ and $B=b_{1} \cdots b_{n-1}$ such that $f(A 0) \neq f(A 1)$ and $g(B 0) \neq g(B 1)$. By $(2.5), f(0 C) \neq f(1 C)$ for every $(m-1)$ block $C$; thus, given an $(m-1)$-block $C$ and $b=0$ or 1 , we can choose $c=0$ or 1 such that $f(c C)=b$. Therefore we may successively choose $c_{n-1}, c_{n-2}, \ldots, c_{1}$ such that

(1) if $n \leqslant m$, then

$$
f\left(c_{n-1} a_{1} \cdots a_{m-1}\right)=b_{n-1}, \ldots, f\left(c_{1} \cdots c_{n-1} a_{1} \cdots a_{m-n+1}\right)=b_{1},
$$

(2) if $n>m$, then

$$
f\left(c_{n-1} a_{1} \cdots a_{m-1}\right)=b_{n-1}, \ldots, f\left(c_{n-m+1} \cdots c_{n-1} a_{1}\right)=b_{n-m+1}
$$

and

$$
f\left(c_{n-m} \cdots c_{n-1}\right)=b_{n-m}, \ldots, f\left(c_{1} \cdots c_{m}\right)=b_{1} .
$$

Let $C=c_{1} \cdots c_{n-1}$. Then either

$$
g \circ f(C A 0)=g(B 0) \text { and } g \circ f(C A 1)=g(B 1)
$$

or

$$
g \circ f(C A 0)=g(B 1) \text { and } g \circ f(C A 1)=g(B 0) \text {. }
$$

Since $g(B 0) \neq g(B 1)$, in either case we have $g \circ f(C A 0) \neq g \circ f(C A 1)$. Therefore $\rho(g \circ f)=n+m-1$.

We remark that (2.10) need not hold if $f$ is not linear in the first variable. Let $f \in \mathscr{F}_{4}$ where

$$
f\left(x_{1} x_{2} x_{3} x_{4}\right)=x_{2}+x_{1} x_{4}+x_{1} x_{3} x_{4} .
$$

Then $f \circ f \in \mathscr{F}_{7}$ and

$$
f \circ f\left(x_{1} \cdots x_{7}\right)=x_{3} .
$$

Thus $\rho(f)=4$ and $\rho(f \circ f)=3$.

The following theorem is an immediate consequence of (2.5) and (2.10).

(2.11) TheOrem (Ferguson). If $f \in \mathscr{L}_{1}$ is nontrivial and $g \circ f=g$, then $g$ is constant.

(2.12) THEOREM. If $f \in \mathscr{L}_{1}$ is nontrivial, then $\mathcal{C}^{*}(f) \subseteq \mathscr{L}_{1}$. 
Proof. Let $g \in \mathcal{C}^{*}(f)$. Since $Q g \circ f=Q g, Q g$ is constant by Ferguson's Theorem. If $Q g=0$, then $\lambda(g) \neq 1$, therefore $Q g=1$, i.e., $g \in \mathfrak{L}_{1}$.

We conclude this section with two results which will be useful in the solution of the commuting block maps problem for the class of block maps considered in $\$ \S 4-6$.

(2.13) Lemma. If $f \in \mathscr{L}_{1}$ and $g \circ f=h \circ f$, then $g=h$.

Proof. It suffices to show that if $g \circ f=0$, then $g=0$. Suppose $g$ is not constant, i.e., $\rho(g) \geqslant 1$. Since $\rho(f) \geqslant 1$, by $(2.10)$ we have

$$
\rho(g \circ f)=\rho(g)+\rho(f)-1 \geqslant 1 .
$$

Therefore $g$ is constant. Since $1 \circ f=1$, it follows that $g=0$.

(2.14) Lemma. Let $f \in \mathscr{L}_{1}$ and let $n \geqslant 0$. Then $g \in \mathcal{C}(f)$ if and only if $g \circ f^{n} \in \mathcal{C}(f)$.

Proof. The "only if" statement is clear. Suppose then that $g \circ f^{n} \in \mathcal{C}(f)$. Then $(g \circ f) \circ f^{n}=(f \circ g) \circ f^{n}$. Since $f^{n} \in \mathcal{L}_{1}, g \circ f=f \circ g$ by (2.13).

3. Determination of $e(f)$ when $f$ is linear. In this section we will be concerned with block maps of the form $f\left(x_{1} \cdots x_{n}\right)=a_{0}+\sum a_{i} x_{i}$, which we will call linear. We will solve the commuting block maps problem for linear block maps.

Notation. Whenever the summation symbol $\Sigma$ appears in this section without limits, the lower limit will be assumed to be 1 and the upper limit will be assumed to be finite.

A block map $f$ is called linear, written $f \in \mathcal{L}$, provided it is a finite sum of the form

$$
a_{0}+\sum a_{i} T^{i-1} I
$$

where $a_{i}=0$ or 1 . In terms of polynomials, an $n$-block map $f$ is linear if and only if there exist $a_{0}, a_{1}, \ldots, a_{n}=0$ or 1 such that

$$
f\left(x_{1} \cdots x_{n}\right)=a_{0}+\sum_{i=1}^{n} a_{i} x_{i} \text {. }
$$

The nontrivial members of $\mathcal{E}$ are easily recognized: $a_{0}+\sum a_{i} T^{i-1} I$ is nontrivial if and only if $\operatorname{card}\left\{i \geqslant 1 \mid a_{i}=1\right\} \geqslant 2$.

Let $f=a_{0}+\sum a_{i} T^{i-1} I$ be linear. We say that $f$ is homogeneous, written $f \in \mathfrak{L}_{H}$, or inhomogeneous, written $f \in \mathfrak{L}_{I}$, according as $a_{0}=0$ or 1 .

We noted in $\S 1$ that with operations + and $\circ$ the set of all block maps satisfies all the axioms for a ring except one of the distributive laws. However, $\mathcal{L}_{H}$ is a ring, in fact it is a unique factorization domain.

(3.1) Theorem [F, p. 53]. With operations + and $\circ, \mathfrak{L}_{H}$ is isomorphic to $\mathbf{Z}_{2}[x]$, the ring of polynomials in one indeterminate over $\mathbf{Z}_{2}$. 
PROof. The isomorphism is given by

$$
\sum a_{i} T^{i-1} I \rightarrow \sum a_{i} x^{i-1} \text {. }
$$

We note the fact, first observed by Ferguson [F, Theorem 3.3], that $\mathfrak{L}_{H}$ is characterized by its distributivity properties: $f \in \mathfrak{L}_{H}$ if and only if $f \circ(g+$ $h)=f \circ g+f \circ h$ for all $g, h$.

An immediate consequence of (3.1) is

(3.2) Lemma. If $f \in \mathfrak{L}_{H}$, then $\mathfrak{L}_{H} \subseteq \mathcal{C}(f)$.

(3.3) Lemma. If $f \in \mathcal{L}$ is nontrivial, then $\mathcal{C}(f) \subseteq \mathcal{L}$.

Proof. Let $f=a_{0}+\sum a_{i} T^{i-1} I$. Since $f$ is not constant, $a_{i}=1$ for some $i \geqslant 1$. If $\lambda(f)>1$, i.e., if $a_{1}=0$, then $f=T^{\lambda(f)-1} h$ where $\lambda(h)=1$. Since $f \in \mathcal{L}$, so is $h$, and $\mathcal{C}(f)=\mathcal{C}(h)$. Therefore we may assume that $\lambda(f)=1$, $a_{1}=1$, in particular $f \in \mathscr{L}_{1}$.

We will show by induction on $\rho(g)$ that

$$
\text { if } g \circ f+f \circ g \text { is constant, then } g \in \mathcal{L} \text {. }
$$

Let $\rho(g)=0$. Then $g$ is constant and so $g \in \mathfrak{L}$.

Suppose $n \geqslant 1$ and (*) is true for all block maps with right extent less than $n$. Let $g \circ f+f \circ g$ be constant and let $\rho(g)=n$. Then $Q(g \circ f+f \circ g)=0$ and so $Q(g \circ f)=Q(f \circ g)$. But $Q(g \circ f)=Q g \circ f$ and $Q(f \circ g)=Q g$. Therefore by Ferguson's Theorem (2.11), $Q g$ is constant, call it $b$.

We now have $g=b I+T R g$ and $\rho(R g)<n$. Thus

$$
\begin{aligned}
& g \circ f=b\left(a_{0}+\sum a_{i} T^{i-1} I\right)+T R g \circ f, \\
& f \circ g=b \sum a_{i} T^{i-1} I+f \circ T R g,
\end{aligned}
$$

and so

$$
g \circ f+f \circ g=a_{0} b+T(R g \circ f+f \circ R g) .
$$

Therefore $T(R g \circ f+f \circ R g)$ is constant and hence so is $R g \circ f+f \circ R g$. By the inductive hypothesis, $R g \in \mathcal{L}$. Therefore $g \in \mathcal{L}$.

Let $f=a_{0}+\sum a_{i} T^{i-1} I$ be linear. We say that $f$ is even or odd according as $\operatorname{card}\left\{i \geqslant 1 \mid a_{i}=1\right\}$ is even or odd.

(3.4) Lemma. Let $f \in \mathcal{L}_{H}$ and $g, h \in \mathcal{L}_{I}$. Then

(a) $g \circ f=f \circ g$ if and only if $f$ is odd.

(b) $h \circ g=g \circ h$ if and only if $g$ and $h$ are either both even or both odd.

Proof. Let

$$
f=\sum a_{i} T^{i-1} I, \quad g=1+\sum b_{i} T^{i-1} I
$$

and

$$
h=1+\sum c_{i} T^{i-1} I
$$


Then

$$
\begin{gathered}
g \circ f=1+\sum \sum a_{i} b_{j} T^{i+j-2} I \text { and } \\
f \circ g=\sum a_{i}+\sum \sum a_{i} b_{j} T^{i+j-2} I
\end{gathered}
$$

Therefore $g \circ f=f \circ g$ if and only if $\sum a_{i}=1$, i.e., $f$ is odd.

Similarly,

$$
g \circ h=1+\sum b_{i}+\sum \sum b_{i} c_{j} T^{i+j-2} I
$$

and

$$
h \circ g=1+\sum c_{j}+\sum \sum b_{i} c_{j} T^{i+j-2} I
$$

Therefore $h \circ g=g \circ h$ if and only if $\sum b_{i}=\sum c_{j}$, i.e., $g$ and $h$ are either both even or both odd.

The solution to the commuting block maps problem for nontrivial $f \in \mathcal{E}$ now follows from the preceding results.

(3.5) THEOREM. Let $f \in \mathcal{E}$ be nontrivial. Then

(a) If $f \in \mathcal{L}_{H}$ and $f$ is odd, then $\mathcal{C}(f)=\mathcal{L}$.

(b) If $f \in \mathcal{L}_{H}$ and $f$ is even, then $\mathcal{C}(f)=\mathcal{L}_{H}$.

(c) If $f \in \mathcal{L}_{I}$ and $f$ is odd, then $\mathcal{C}(f)=\{g \in \mathcal{L} \mid g$ is odd $\}$.

(d) If $f \in \mathcal{L}_{I}$ and $f$ is even, then

$$
\mathcal{C}(f)=\left\{g \in \mathcal{L}_{H} \mid g \text { is odd }\right\} \cup\left\{g \in \mathcal{L}_{I} \mid g \text { is even }\right\}
$$

We now turn our attention to nonconstant, trivial block maps, i.e., those of the form $T^{n} I$ or $T^{n} I+1$. By (1.2), we may assume that $n=0$. Since $I$ commutes with all block maps, we need only consider $f=I+1$.

For $b=0$ or 1 , let $\bar{b}=1+b$. Thus $\overline{0}=1$ and $\overline{1}=0$. For an $n$-block $B=b_{1} \cdots b_{n}$, let $\bar{B}=\bar{b}_{1} \cdots \bar{b}_{n}$.

(3.6) Lemma. Let $f \in \mathcal{F}_{n}$. Then $f \in \mathcal{C}(I+1)$ if and only if $f(B)+f(\bar{B})=1$ for every $n$-block $B$.

We define the skew-translation operator $S$ on the set of block maps as follows. If $f \in \mathscr{F}_{n}$, then $S f \in \mathscr{F}_{n+1}$ is defined by

$$
S f(0 B)=f(B), \quad S f(1 B)=f(\bar{B})+1
$$

(3.7) Proposition. $\mathcal{C}(I+1)=\{S f \mid f \in \mathcal{F}\}$. 
Proof. Let $f \in \mathscr{F}_{n}$. Then for every $n$-block $B$,

$$
\begin{aligned}
& S f(0 B)+S f(\overline{0 B})=S f(0 B)+S f(1 \bar{B})=f(B)+f(\overline{\bar{B}})+1=1, \\
& S f(1 B)+S f(\overline{1 B})=S f(1 B)+S f(0 \bar{B})=f(\bar{B})+1+f(\bar{B})=1,
\end{aligned}
$$

and so $S f \in \mathcal{C}(I+1)$.

Let $f \in \mathcal{C}(I+1)$. We may assume that $f \in \mathscr{F}_{n}$ where $n \geqslant 2$. Define $g \in \mathscr{F}_{n-1}$ by $g(B)=f(0 B)$. Since $f(1 B)+f(\overline{1 B})=1$,

$$
f(1 B)=f(\overline{1 B})+1=f(0 \bar{B})+1=g(\bar{B})+1 .
$$

Therefore $f=S g$.

The solution of the commuting block maps problem for linear block maps is completed by the following result. Recall that if $a=0$ or 1 , then $a^{n}$ denotes the $n$-block consisting entirely of $a$ 's.

(3.8) THEOREM. Let $f$ be linear and trivial.

(1) If $f$ is constant, then $\mathcal{C}(f)=\left\{g \in \mathscr{F}_{n} \mid n \geqslant 1, g\left(a^{n}\right)=a\right\}$, where $f$ is the constant map a.

(2) If $f$ is not constant and $f \in \mathfrak{E}_{H}$, then $\mathcal{C}(f)=\mathscr{F}$.

(3) If $f$ is not constant and $f \in \mathcal{L}_{I}$, then $\mathcal{C}(f)=\{S g \mid g \in \mathscr{F}\}$.

4. The Sums of Powers Theorem. In this section we show that if $f \in \mathscr{F}_{k+1}$ is given by

$$
f\left(x_{0} \cdots x_{k}\right)=x_{0}+\varphi\left(x_{1} \cdots x_{k}\right)
$$

where $k \geqslant 2$ and the full product $x_{1} x_{2} \cdots x_{k}$ is a summand of $\varphi\left(x_{1} \cdots x_{k}\right)$, then every member of $\mathcal{C}^{*}(f)$ is the sum of an odd number of powers of $f$. We note that $x_{1} x_{2} \cdots x_{k}$ is a summand of $\varphi\left(x_{1} \cdots x_{k}\right)$ if and only if $Q^{k} \varphi=1$. Furthermore, this condition holds if and only if the number of $k$-blocks $B$ such that $\varphi(B)=0$ is odd. We note that for each $k \geqslant 0$ exactly half the $(k+1)$-block maps linear in the first variable satisfy this condition.

We will prove the Sums of Powers Theorem by defining an operator $L$ on the set of block maps such that (1) if $g \in e^{*}(f)$, then $L(R g)=0$ and (2) if $L \psi=0$, then $\psi$ is a sum of remainders of powers of $f$. The result then follows because $T R f^{n}=f^{0}+f^{n}$.

The operator $L$ is derived in the following manner. Suppose $g \in \mathcal{C}^{*}(f)$ is an $(n+1)$-block map. We "expand" the equation

$$
g \circ f\left(x_{0} \cdots x_{n+k}\right)+f \circ g\left(x_{0} \cdots x_{n+k}\right)=0
$$

and set the "coefficient" of $x_{1} x_{2} \cdots x_{k-1}$ equal to zero. In formal terms, $L \psi=Q^{k-1}(g \circ f+f \circ g)$ where $g=I+T \psi$.

For example, let $f\left(x_{0} x_{1} x_{2} x_{3}\right)=x_{0}+x_{1} x_{2} x_{3}$ and let $g \in e^{*}(f)$ with $g\left(x_{0} \ldots x_{n}\right)=x_{0}+\psi\left(x_{1} \cdots x_{n}\right)$. Then 


$$
\begin{aligned}
g \circ f & \left(x_{0} \cdots x_{n+3}\right)+f \circ g\left(x_{0} \cdots x_{n+3}\right) \\
= & \left(x_{0}+x_{1} x_{2} x_{3}\right)+\psi \circ f\left(x_{1} \cdots x_{n+3}\right)+\left[x_{0}+\psi\left(x_{1} \cdots x_{n}\right)\right] \\
& +\left[x_{1}+\psi\left(x_{2} \cdots x_{n+1}\right)\right]\left[x_{2}+\psi\left(x_{3} \cdots x_{n+2}\right)\right]\left[x_{3}+\psi\left(x_{4} \cdots x_{n+3}\right)\right] \\
= & \psi \circ f\left(x_{1} \cdots x_{n+3}\right)+\psi\left(x_{1} \cdots x_{n}\right)+x_{1} x_{2} \psi\left(x_{4} \cdots x_{n+3}\right) \\
& +x_{1} x_{3} \psi\left(x_{3} \cdots x_{n+2}\right)+x_{1} \psi\left(x_{3} \cdots x_{n+2}\right) \psi\left(x_{4} \cdots x_{n+3}\right) \\
& +x_{2} x_{3} \psi\left(x_{2} \cdots x_{n+1}\right)+x_{2} \psi\left(x_{2} \cdots x_{n+1}\right) \psi\left(x_{4} \cdots x_{n+3}\right) \\
& +x_{3} \psi\left(x_{2} \cdots x_{n+1}\right) \psi\left(x_{3} \cdots x_{n+2}\right) \\
& +\psi\left(x_{2} \cdots x_{n+1}\right) \psi\left(x_{3} \cdots x_{n+2}\right) \psi\left(x_{4} \cdots x_{n+3}\right) .
\end{aligned}
$$

Therefore the coefficient of $x_{1} x_{2}$ in this expression is

$$
\begin{aligned}
& \psi\left(x_{4} \cdots x_{n+3}\right)+\left[\text { coefficient of } x_{1} x_{2} \text { in } \psi\left(x_{1} \cdots x_{n}\right)\right] \\
& +\left[\text { coefficient of } x_{1} x_{2} \text { in } \psi \circ f\left(x_{1} \cdots x_{n+3}\right)\right] .
\end{aligned}
$$

The lack of economy of the "polynomial" approach should now be clear. For the remainder of this section, we proceed formally.

Notation. For the remainder of this section, $f=I+T \varphi$ where $\rho(\varphi)=k$ $\geqslant 2$ and $Q^{k} \varphi=1$. In particular, $R f=\varphi$.

(4.1) LemMA. If $\rho(\varphi)=k$ and $Q^{k} \varphi=1$, then $Q^{k-1} \varphi=I+b$ for some $b=0$ or 1 .

Proof. By (2.3), $Q^{k-1} \varphi=I \cdot T Q Q^{k-1} \varphi+T R Q^{k-1} \varphi$. Therefore $Q^{k-1} \varphi=$ $I+T R Q^{k-1} \varphi$. By $(2.2), \rho\left(Q^{k-1} \varphi\right) \leqslant \rho(\varphi)-(k-1)=1$, so $\rho\left(Q^{k-1} \varphi\right)=1$. Thus $T R Q^{k-1} \varphi$ is constant and the result is proved.

(4.2) LEMMA. If $g \in e^{*}(f)$, then

$$
Q^{k-1} R g+Q^{k-1} R g \circ f+T R g=0 .
$$

Proof. By (2.12), $g \in \mathcal{L}_{1}$, so we write $g=I+T R g$. Since $f=I+T R f$, we have

$$
g \circ f+f \circ g=T(R g+R g \circ f+R f+R f \circ g) .
$$

Thus

$$
R g+R g \circ f+R f+R f \circ g=0 .
$$

But $Q^{k-1} R f=I+b$, hence

$$
Q^{k-1}(R f+R f \circ g)=(I+b)+(I+b) \circ g=T R g
$$

and so

$$
\begin{aligned}
0 & =Q^{k-1}[R g+R g \circ f+R f+R f \circ g] \\
& =Q^{k-1} R g+Q^{k-1} R g \circ f+T R g . \square
\end{aligned}
$$


We define an operator $L$ on the set of block maps as follows:

$$
L \psi=Q^{k-1} \psi+Q^{k-1} \psi \circ f+T \psi .
$$

The properties of $L$ listed below follow from the definition of $L$ and the properties of $T$ and $Q$ listed earlier.

(4.3) LeMma. (a) $L$ is additive, i.e., $L\left(\psi+\psi^{\prime}\right)=L \psi+L \psi^{\prime}$.

(b) $L 0=0$ and $L 1=1$.

(c) $L(\psi \circ f)=L \psi \circ f$.

(d) $L R f=0$.

(4.4) LemMa. If $L \psi$ is constant, then so is $Q^{k} \psi$.

Proof. Since $L \psi$ is constant, $Q L \psi=0$. Then

$$
Q L \psi=Q^{k} \psi+Q^{k} \psi \circ f .
$$

By Ferguson's Theorem (2.11), $Q^{k} \psi$ is constant.

(4.5) Lemma. Suppose $L \psi$ is constant and $\rho(\psi) \geqslant k$. Then

$$
Q^{k-1} \psi=a I+T \psi^{\prime}
$$

where $a=0$ or $1, L \psi^{\prime}$ is constant, and $\rho\left(\psi^{\prime}\right)=\rho(\psi)-k$.

Proof. Let $R\left(Q^{k-1} \psi\right)=\psi^{\prime}$. Then

$$
Q^{k-1} \psi=I \cdot T Q Q^{k-1} \psi+T \psi^{\prime}=I \cdot T Q^{k} \psi+T \psi^{\prime} .
$$

By (4.4), $Q^{k} \psi$ is constant, call it $a$. Hence

$$
Q^{k-1} \psi=a I+T \psi^{\prime}
$$

Let $L \psi=c$. Then,

$$
\begin{aligned}
c & =\left[a I+T \psi^{\prime}\right]+\left[a(I+T R f)+T \psi^{\prime} \circ f\right]+T \psi \\
& =T\left(\psi^{\prime}+\psi^{\prime} \circ f+a R f+\psi\right),
\end{aligned}
$$

and so

$$
\psi^{\prime}+\psi^{\prime} \circ f+a R f+\psi=c .
$$

Then

$$
L \psi^{\prime}+L \psi^{\prime} \circ f+L \psi=c
$$

and so $L \psi^{\prime}=L \psi^{\prime} \circ f$. By Ferguson's Theorem (2.11), $L \psi^{\prime}$ is constant.

Suppose $\rho\left(\psi^{\prime}\right)=0$. We show $\rho(\psi)=k$. Since $\psi^{\prime}$ is constant, $\psi^{\prime}=\psi^{\prime} \circ f$. Thus (*) becomes $a R f+\psi=c$, and so $\rho(a R f+\psi)=0$. Now $\rho(\psi)>0$, so by (1.3), $\rho(\psi)=\rho(a R f)$. Hence $a=1$ and so $\rho(\psi)=\rho(R f)=k$.

Suppose $\rho\left(\psi^{\prime}\right)>0$. Write (*) as

$$
\psi^{\prime}+\psi^{\prime} \circ f=a R f+\psi+c,
$$

and so

$$
\rho\left(\psi^{\prime}+\psi^{\prime} \circ f\right)=\rho(a R f+\psi+c) .
$$


Since $\psi^{\prime}$ is not constant, it follows from (2.10) that $\rho\left(\psi^{\prime} \circ f\right)=\rho\left(\psi^{\prime}\right)+k$ and so $\rho\left(\psi^{\prime}+\psi^{\prime} \circ f\right)=\rho\left(\psi^{\prime}\right)+k$. If $\rho(\psi)=k$, then $\rho(\psi) \geqslant \rho(a R f) \geqslant \rho(c)=0$ and so $\rho(a R f+\psi+c) \leqslant \rho(\psi)=k$. Therefore $\rho\left(\psi^{\prime}\right)+k \leqslant k$, which implies that $\rho\left(\psi^{\prime}\right)=0$. Thus $\rho(\psi)>k$, and so $\rho(\psi)>\rho(a R f) \geqslant \rho(c)$. Then $\rho(a R f+\psi$ $+c)=\rho(\psi)$. Hence $\rho\left(\psi^{\prime}\right)+k=\rho(\psi)$.

(4.6) LeMMA. If $L \psi$ is constant, then $\rho(\psi)=n k$ for some $n \geqslant 0$.

Proof. Let $\rho(\psi)=n k+r$ where $n \geqslant 0$ and $0 \leqslant r \leqslant k-1$. We show that $r=0$ by induction on $n$.

Let $n=0$. Then $\rho(\psi) \leqslant k-1$ and so by repeated application of (2.2), $\rho\left(Q^{k-1} \psi\right)=0$. Therefore $Q^{k-1} \psi$ is constant and so

$$
Q^{k-1} \psi+Q^{k-1} \psi \circ f=0 .
$$

Hence $L \psi=T \psi$ and so $T \psi$ is constant. Therefore $\rho(\psi)=0$.

Let $n \geqslant 1$ and suppose the lemma is true for all nonnegative integers less than $n$. Let $L \psi$ be constant and $\rho(\psi)=n k+r$. Then $Q^{k-1} \psi=a I+T \psi^{\prime}$ where $\rho\left(\psi^{\prime}\right)=(n-1) k+r$ and $L \psi^{\prime}$ is constant. By the inductive hypothesis, $r=0$.

Suppose $L \psi$ is constant. We associate with $\psi$ a sequence $a_{i}=a_{i}(\psi), i \geqslant 0$, defined by repeated application of (4.5). Specifically, let $\psi_{0}=\psi$ and for $n \geqslant 0, Q^{k-1} \psi_{n}=a_{n} I+T \psi_{n+1}$.

It follows from (4.5) that if $L \psi$ is constant and $\rho(\psi)=n k$, then $a_{i}(\psi)=0$ and $\psi_{i}=0$ for all $i \geqslant n$.

The next result is an immediate consequence of the additivity of $T, Q$ and $L$.

(4.7) LemMa. If $L \psi$ and $L \psi^{\prime}$ are constant, then so is $L\left(\psi+\psi^{\prime}\right)$. Furthermore $a_{i}\left(\psi+\psi^{\prime}\right)=a_{i}(\psi)+a_{i}\left(\psi^{\prime}\right)$ for all $i \geqslant 0$.

(4.8) LEMMA. If $L \psi$ is constant and $\rho(\psi)=n k$, where $n \geqslant 1$, then $a_{n-1}(\psi)=$ 1 .

Proof. We prove the lemma by induction on $n$. Recall that $Q^{k-1} \psi=a_{0} I$ $+T \psi_{1}$.

Let $n=1$, i.e., $\rho(\psi)=k$. Then by (4.5), $\rho\left(\psi_{1}\right)=0$ and so $\psi_{1}$ is constant, say $b$. Therefore $Q^{k-1} \psi=a_{0} I+b$ and so

$$
L \psi=a_{0} T R f+T \psi .
$$

If $a_{0}=0$, then $L \psi=T \psi$ is not constant. Therefore $a_{0}=1$.

Suppose that $n \geqslant 2$ and the lemma is true for $n-1$. If $L \psi$ is constant and $\rho(\psi)=n k$, then by $(4.5), L \psi_{1}$ is constant and $\rho\left(\psi_{1}\right)=(n-1) k$. By the inductive hypothesis, $a_{n-2}\left(\psi_{1}\right)=1$. But $a_{i}\left(\psi_{1}\right)=a_{i+1}(\psi)$ for all $i \geqslant 0$. Therefore $a_{n-1}(\psi)=1$. 
(4.9) Lemma. Let $L \psi=0$ and $\rho(\psi)=n k$ where $n \geqslant 1$. Let $\psi^{\prime}=\psi+R f^{n}$. Then $L \psi^{\prime}=0$ and $\rho\left(\psi^{\prime}\right)=m k$ where $0 \leqslant m \leqslant n-1$.

Proof. Since $f^{n}=I+T R f^{n} \in \mathcal{C}(f), L R f^{n}=0$. Since $L$ is additive, $L \psi^{\prime}=$ 0 . Hence $\rho\left(\psi^{\prime}\right)=m k$ for some $m \geqslant 0$. Since $\rho\left(R f^{n}\right)=n k$, it follows from (1.3) that $0 \leqslant m \leqslant n$.

For all $i \geqslant 0, a_{i}\left(\psi^{\prime}\right)=a_{i}(\psi)+a_{i}\left(R f^{n}\right)$, and since $\rho(\psi)=\rho\left(R f^{n}\right)=n k$, $a_{n-1}(\psi)=a_{n-1}\left(R f^{n}\right)=1$. Therefore $a_{n-1}\left(\psi^{\prime}\right)=0$ and so by (4.8), $\rho\left(\psi^{\prime}\right) \neq n k$.

Therefore $0 \leqslant m \leqslant n-1$.

(4.10) LEMMA, If $L \psi=0$, then $\psi$ is a sum of remainders of powers of $f$.

Proof. By (4.6), $\rho(\psi)=n k$ for some $n \geqslant 0$. We prove the lemma by induction on $n$.

Let $n=0$. Then $\psi=0$ is the empty sum. Suppose $n \geqslant 1$ and the lemma is true for all nonnegative integers less than $n$. Let $L \psi=0$ and $\rho(\psi)=n k$. Let $\psi^{\prime}=\psi+R f^{n}$. Then $L \psi^{\prime}=0$ and $\rho\left(\psi^{\prime}\right)=m k$ where $0 \leqslant m \leqslant n-1$. By the inductive hypothesis, $\psi^{\prime}$ is a sum of remainders of powers of $f$, and therefore so is $\psi=\psi^{\prime}+R f^{n}$.

(4.11) THeOREM. Let $f\left(x_{0} \cdots x_{k}\right)=x_{0}+\varphi\left(x_{1} \cdots x_{k}\right)$ where $k \geqslant 2$ and the full product $x_{1} x_{2} \cdots x_{k}$ is a summand of $\varphi\left(x_{1} \cdots x_{k}\right)$. Then every member of $\mathcal{C}^{*}(f)$ is the sum of an odd number of powers of $f$.

Proof. Let $g \in \mathcal{C}^{*}(f)$. Then $g=f^{0}+T R g$ and $L R g=0$. By (4.10), $R g$ is a sum of remainders of powers of $f$. Since $T R f^{n}=f^{0}+f^{n}$, if $R g$ is the sum of $m$ remainders of powers of $f$, then $g$ is the sum of $(2 m+1)$ powers of $f$.

5. The Small Period Theorem. In this section we will determine $e^{*}(f)$ when

$$
f\left(x_{0} \cdots x_{k}\right)=x_{0}+\prod_{i=1}^{k}\left(x_{i}+\delta_{i}\right)
$$

where $k \geqslant 2$ and the least period of $\delta_{1} \cdots \delta_{k}$ is no greater than $\frac{1}{2} k$.

By (4.11), any member of $e^{*}(f)$ is the sum of an odd number of powers of $f$. We show that if $g$ is the sum of $2 s+1$ distinct powers of $f$ with $s>0$, then there exists $x \in X$ such that $g_{\infty} \circ f_{\infty}(x) \neq f_{\infty} \circ g_{\infty}(x)$. This implies that $e^{*}(f)=\left\{f^{n} \mid n \geqslant 0\right\}$.

Let $B=b_{1} \cdots b_{n}$ be an $n$-block and let $1 \leqslant p \leqslant n$. We say that $p$ is a period of $B$ provided $b_{i}=b_{i+p}$ for $1 \leqslant i \leqslant n-p$. Note that $n$ is a period of every $n$-block.

Notation. Throughout this section,

(1) $f=I+\prod_{i=1}^{k} T^{i}\left(I+\delta_{i}\right)$ where $k \geqslant 2$.

(2) The least period of $\delta_{1} \cdots \delta_{k}$ is $p$.

(3) $y, z \in X$ are defined as follows. 


$$
\begin{aligned}
& y_{n p}=1+\delta_{p} \text { and } y_{n p+r}=1+\delta_{r} \text { for } 1 \leqslant r \leqslant p-1 . \\
& z_{n p}=\delta_{p} \text { and } z_{n p+r}=1+\delta_{r} \text { for } 1 \leqslant r \leqslant p-1 .
\end{aligned}
$$

(5.1) Proposition. $f_{\infty}(y)=z$.

PROof. It suffices to show that $\left[f_{\infty}(y)\right]_{n p}=1+y_{n p}$ and $\left[f_{\infty}(y)\right]_{n p+r}=$ $y_{n p+r}$ for $1 \leqslant r \leqslant p-1$.

The first equality follows from the fact that $y_{i}=y_{i+p}$ for all $i \in \mathbf{Z}$.

Suppose that $1 \leqslant r \leqslant p-1$. Then

$$
\left[f_{\infty}(y)\right]_{n p+r}=y_{n p+r}+\prod_{i=1}^{k}\left(y_{r+i}+\delta_{i}\right) .
$$

If $y_{r+i}=1+\delta_{i}$ for $1 \leqslant i \leqslant k$, then in particular, $y_{j+r}=1+\delta_{j}$ for $1<j<k$ $-r$. However $y_{j+r}=1+\delta_{j+r}$ for $1 \leqslant j \leqslant k-r$, so $\delta_{j}=\delta_{j+r}$ for $1 \leqslant j \leqslant k$ - $r$. Therefore $r$ is a period of $\delta_{1} \cdots \delta_{k}$, contradicting the minimality of $p$. Therefore $y_{r+i}=\delta_{i}$ for some $i, 1 \leqslant i \leqslant k$, and so $\left[f_{\infty}(y)\right]_{n p+r}=y_{n p+r}$.

(5.2) Proposition. $f_{\infty}(z)=z$.

Proof. Let $i \in \mathbf{Z}$. Since $\sum_{j=1}^{p} z_{i+j}=1+\sum_{j=1}^{p}\left(1+\delta_{j}\right), z_{i+j}=\delta_{j}$ for some $j$, $i \leqslant j \leqslant p$. Hence $\Pi_{j=1}^{k}\left(z_{i+j}+\delta_{j}\right)=0$ and so $\left[f_{\infty}(z)\right]_{i}=z_{i}$.

(5.3) THeOREM. Let $f\left(x_{0} \cdots x_{k}\right)=x_{0}+\Pi_{i=1}^{k}\left(x_{i}+\delta_{i}\right)$ where $k \geqslant 2$. Let $p$ be the least period of $\delta_{1} \cdots \delta_{k}$. If $p \leqslant \frac{1}{2} k$, then $\mathcal{C}^{*}(f)=\left\{f^{n} \mid n \geqslant 0\right\}$.

Proof. It suffices to show that $\mathcal{C}^{*}(f) \subseteq\left\{f^{n} \mid n \geqslant 0\right\}$. Let $g \in \mathcal{C}^{*}(f)$. By (4.11), $g=\sum_{i=0}^{2 s} f^{m_{i}}$ where $s \geqslant 0$. Without loss of generality, we may assume that $0 \leqslant m_{0}<\cdots<m_{2 s}$. We show that $s=0$.

Suppose $s \geqslant 1$. By (2.14) we may assume that $m_{0}=0$. Since $f \in \mathscr{L}_{1}$, so is $f^{m_{1}}$ and hence $f_{\infty}^{m_{1}}$ maps $X$ onto $X[\mathbf{H}$, Theorem 6.6]. Therefore there exists $x \in X$ such that $f_{\infty}^{m_{1}}(x)=y$. Since

$$
g_{\infty} \circ f_{\infty}=\sum_{i=0}^{2 s} f_{\infty}^{m_{i}+1}
$$

and $f_{\infty}^{m}(x)=z$ for $m>m_{1}$

$$
\sum_{i=1}^{2 s} f_{\infty}^{m_{i}+1}(x)=w
$$

where $w_{j}=0$ for all $j \in \mathbf{Z}$ and

$$
\sum_{i=2}^{2 s} f_{\infty}^{m_{i}}(x)=z .
$$

It follows that

$$
g_{\infty} \circ f_{\infty}(x)=f_{\infty}(x)
$$


and

$$
g_{\infty}(x)=x+y+z .
$$

Let $q$ be the largest integer such that $q p \leqslant k$. Since $2 p \leqslant k, q \geqslant 2$. Then

$$
\left[g_{\infty} \circ f_{\infty}(x)\right]_{n p}=\left[f_{\infty}(x)\right]_{n p}=x_{n p}+\prod_{i=1}^{k}\left(x_{n p+i}+\delta_{i}\right) .
$$

Since $(y+z)_{n p}=1$ and $(y+z)_{n p+r}=0$ for $1 \leqslant r \leqslant p-1,(x+y+$ $z)_{n p}=1+x_{n p}$ and $(x+y+z)_{n p+r}=x_{n p+r}$ for $1 \leqslant r \leqslant p-1$.

But $f_{\infty} \circ g_{\infty}(x)=f_{\infty}(x+y+z)$, so

$$
\left[f_{\infty} \circ g_{\infty}(x)\right]_{n p}=1+x_{n p}+\prod_{i \in J}\left(x_{n p+i}+1+\delta_{i}\right) \prod_{i \in J^{\prime}}\left(x_{n p+i}+\delta_{i}\right)
$$

where

$$
J=\{j p \mid 1 \leqslant j \leqslant q\} \quad \text { and } \quad J^{\prime}=\{i \mid 1 \leqslant i \leqslant k, i \notin J\} .
$$

Since $\delta_{i}=\delta_{p}$ for all $i \in J$ and $\left[f_{\infty} \circ g_{\infty}(x)\right]_{n p}+\left[g_{\infty} \circ f_{\infty}(x)\right]_{n p}=0$,

$$
1=\prod_{i=1}^{k}\left(x_{n p+i}+\delta_{i}\right)+\prod_{i \in J}\left(x_{n p+i}+1+\delta_{i}\right) \prod_{i \in J^{\prime}}\left(x_{n p+i}+\delta_{i}\right) .
$$

Therefore, for each $n \in \mathbf{Z}$, one and only one of the following holds:

$$
\begin{gathered}
x_{n p+i}=1+\delta_{i} \text { for } 1 \leqslant i \leqslant k . \\
x_{n p+i}= \begin{cases}\delta_{i} & \text { for } i \in J, \\
1+\delta_{i} & \text { for } i \in J^{\prime} .\end{cases}
\end{gathered}
$$

Suppose (1) holds for some $n$. Then since $2 p \leqslant k$,

$$
x_{(n+1) p+p}=x_{n p+2 p}=1+\delta_{2 p}=1+\delta_{p} .
$$

But $p \in J$, so (2) does not hold for $n+1$. Consequently (1) holds for $n+1$. Similarly,

$$
x_{(n-1) p+p}=x_{n p}=1+\delta_{p}=1+\delta_{2 p} .
$$

But $q \geqslant 2$ and hence (2) does not hold for $n-1$. Therefore (1) holds for $n-1$. It follows that (1) holds for all $n \in \mathbf{Z}$ and hence $x=y$. Then $f^{m_{1}}(x)=z$, which contradicts the choice of $x$. Therefore (1) holds for no $n \in \mathbf{Z}$, i.e., (2) holds for all $n \in \mathbf{Z}$. But then $x=z$ and so $f^{m_{1}}(x)=z$, again contradicting the choice of $x$.

Therefore we conclude that $s=0$.

We remark that $e^{\prime}(f)$ is easily determined for the block maps considered in this section. If $p=1$, then $e^{\prime}(f)$ consists of the constant map $\delta_{1}$ alone. If $p \geqslant 2$, then $\mathcal{C}^{\prime}(f)$ consists of both constant maps 0 and 1 . 
6. The Large Period Theorem. In this section we will determine $e^{*}(f)$ when

$$
f\left(x_{0} \cdots x_{k}\right)=x_{0}+\prod_{i=1}^{k}\left(x_{i}+\delta_{i}\right)
$$

where $k \geqslant 2$ and the least period of $\delta_{1} \cdots \delta_{k}$ is greater than $\frac{1}{2} k$.

We begin with two technical results about periods of finite blocks.

(6.1) LeMma. Let $p \geqslant 2$ and $1 \leqslant r \leqslant p-1$. If $r$ and $p$ are periods of $b_{1} \cdots b_{p+r-1}$, then $b_{r}=b_{p}$.

PROOF. The proof is by induction on $r$.

If $r=1$, then $b_{1}=b_{2}=\cdots=b_{p}$.

Suppose $2 \leqslant r \leqslant p-1$ and the lemma is true for all positive integers less than $r$. Let $p=n r+s$ where $0 \leqslant s \leqslant r-1$. If $s=0$, then $p=n r$ and so $b_{p}=b_{r}$. If $1 \leqslant s \leqslant r-1$, then $b_{s}=b_{p}$. Furthermore, for $1 \leqslant i \leqslant r-1$, we have

$$
b_{i}=b_{i+p}=b_{i+n r+s}=b_{i+s} .
$$

Therefore $s$ and $r$ are periods of $b_{1} \cdots b_{r+s-1}$. By the inductive hypothesis, $b_{s}=b_{r}$ and thus $b_{r}=b_{p}$.

(6.2) LemMA. Let $2 \leqslant p \leqslant k$ and $1 \leqslant r \leqslant p-1$. If $r$ is a period of $b_{1} \cdots b_{p+r-1}$ and $p$ is a period of $b_{1} \cdots b_{k}$, then $r$ is a period of $b_{1} \cdots b_{k}$.

Proof. If $k \leqslant p+r-1$, then there is nothing to prove. Suppose $k \geqslant p+$ $r$. We show that $b_{i}=b_{i+r}$ for $1 \leqslant i \leqslant k-r$.

Let $1 \leqslant i \leqslant p-1$. Then $b_{i}=b_{i+r}$ since $r$ is a period of $b_{1} \cdots b_{p+r-1}$.

Since $p+r \leqslant k, p$ is a period of $b_{1} \cdots b_{p+r}$ and thus $b_{r}=b_{p+r}$. But $p$ is also a period of $b_{1} \cdots b_{p+r-1}$ and hence by (6.1), $b_{p}=b_{r}$. Therefore $b_{i}=$ $b_{i+r}$ for $i=p$.

Let $p+1 \leqslant i \leqslant k+r$. Then $1 \leqslant i-n p \leqslant p$ for some $n \geqslant 1$. Since $b_{j}=$ $b_{j+r}$ for $1 \leqslant j \leqslant p$, we have $b_{i-n p}=b_{i-n p+r}$. Since $p$ is a period of $b_{1} \cdots b_{k}$, we have $b_{i-n p}=b_{i}$ and $b_{i-n p+r}=b_{i+r}$. Therefore, $b_{i}=b_{i+r}$.

In showing that every sum of an odd number of powers of $f$ commutes with $f$ we will have to "evaluate" expressions such as

$$
R f \circ\left[I+T\left(R f^{n_{1}}+\cdots+R f^{n_{2 s}}\right)\right],
$$

which is the same as "expanding"

$$
\prod_{i=1}^{k} T^{i-1}\left[\left(I+\delta_{i}\right)+T\left(R f^{n_{1}}+\cdots+R f^{n_{2 s}}\right)\right] .
$$

We will write $\alpha_{i}=T^{i-1}\left(I+\delta_{i}\right)$ and $\beta_{i}=T^{i}\left(R f^{n_{1}}+\cdots+R f^{n_{2 s}}\right)$. The above product then is $\prod_{i=1}^{k}\left(\alpha_{i}+\beta_{i}\right)$, and $\alpha_{i}, \beta_{i}$ are elements of $\mathscr{F}$, which is a commutative ring with identity (with operations + and $\cdot$ ). 
We will derive some "orthogonality" relations involving the $\alpha$ 's and $\beta$ 's which will simplify the expansion of the product. We first indicate the consequences of the orthogonality relations.

(6.3) LEMMA. Let $A$ be a commutative ring with identity. Let $k \geqslant 2$ and let $\alpha_{i}, \beta_{j} \in A$ for $1 \leqslant i \leqslant k$ and $j \geqslant 1$. Let $2 \leqslant p \leqslant k$ and for $1 \leqslant r \leqslant p-1$, let $m_{r}=\min \{p-1, k-r\}$. If

(1) $\beta_{j} \beta_{j+r}=0$ for $j \geqslant 1$ and $1 \leqslant r \leqslant p-1$,

(2) $\beta_{r} \prod_{i=1}^{m_{r}} \alpha_{i+r}=0$ for $1 \leqslant r \leqslant p-1$, then

$$
\prod_{i=1}^{k}\left(\alpha_{i}+\beta_{i}\right)=\prod_{i=1}^{p-1} \alpha_{i} \prod_{i=p}^{k}\left(\alpha_{i}+\beta_{i}\right) .
$$

If furthermore, $p>\frac{1}{2} k$, then

$$
\prod_{i=1}^{k}\left(\alpha_{i}+\beta_{i}\right)=\prod_{i=1}^{k} \alpha_{i}+\sum_{j=1}^{k} \beta_{j} \prod_{\substack{i=1 \\ i \neq j}}^{k} \alpha_{i} .
$$

We omit the straightforward but tedious proof.

NotATION. For the remainder of this section,

(1) $f=I+\prod_{i=1}^{k} T^{i}\left(I+\delta_{i}\right)$ where $k \geqslant 2$.

(2) The least period of $\delta_{1} \cdots \delta_{k}$ is $p$ and $p \geqslant 2$.

(3) For $n \geqslant 1, \varphi_{n}=R f^{n}$. Therefore $f^{n}=I+T \varphi_{n}$.

In particular, $\varphi_{1}=\prod_{i=1}^{k} T^{i-1}\left(I+\delta_{i}\right)$.

(4) $\varphi^{\prime}=\Pi_{i=1}^{p-1} T^{i-1}\left(I+\delta_{i}\right)$.

(5) For $1 \leqslant r \leqslant p-1, m_{r}=\min \{p-1, k-r\}$ and

$$
\tilde{\varphi}_{r}=\prod_{i=1}^{m_{r}} T^{i-1}\left(I+\delta_{i+r}\right) .
$$

(6) For $1 \leqslant j \leqslant k$,

$$
\hat{\varphi}_{j}=\prod_{\substack{i=1 \\ i \neq j}}^{k} T^{i-1}\left(I+\delta_{i}\right) .
$$

In terms of polynomials,

$$
\begin{aligned}
& \varphi^{\prime}\left(x_{1} \cdots x_{p-1}\right)=\left(x_{1}+\delta_{1}\right) \cdots\left(x_{p-1}+\delta_{p-1}\right), \\
& \tilde{\varphi}_{r}\left(x_{1} \cdots x_{m_{r}}\right)=\left(x_{1}+\delta_{1+r}\right) \cdots\left(x_{m_{r}}+\delta_{m_{r}+r}\right), \\
& \hat{\varphi}_{j}\left(x_{1} \cdots x_{k}\right)=\left(x_{1}+\delta_{1}\right) \cdots\left(x_{j-1}+\delta_{j-1}\right)\left(x_{j+1}+\delta_{j+1}\right) \cdots\left(x_{k}+\delta_{k}\right) .
\end{aligned}
$$

The main orthogonality results will be that $\varphi_{m} \cdot T^{r} \varphi_{n}=0$ for $1 \leqslant r \leqslant p-$ 1. We will then use (6.3) to show that if $g$ is the sum of an odd number of powers of $f$, then $g \circ f+f \circ g$ can be written as a sum of products of the form $T^{i}\left(\varphi_{m} \cdot T^{r} \varphi_{n}\right)$ where $1 \leqslant r \leqslant p-1$. 
Let $g, h$ be block maps. We say $g$ is a factor of $h$ provided $h=g \cdot h^{\prime}$ for some block map $h^{\prime}$. It follows from the definition that $g$ is a factor of $h$ if and only if $T g$ is a factor of $T h$.

(6.4) Proposition. $\varphi^{\prime}$ is a factor of $\varphi_{1}$, and for $1 \leqslant r \leqslant p-1, T^{r} \tilde{\varphi}_{r}$ is a factor of $\varphi_{1}$.

Proof. It is clear that $\varphi^{\prime}$ is a factor of $\varphi_{1}$.

Suppose $1 \leqslant r \leqslant p-1$. Then

$$
T^{r} \tilde{\varphi}_{r}=\prod_{i=r+1}^{m_{r}+r} T^{i-1}\left(I+\delta_{i}\right)
$$

Since $m_{r}+r \leqslant k, T^{r} \tilde{\varphi}_{r}$ is a factor of $\varphi_{1}$.

(6.5) Proposition. If $1 \leqslant r \leqslant p-1$, then $T^{r} \varphi^{\prime} \cdot T^{r} \tilde{\varphi}_{r}=0$.

Proof. It suffices to show that $\varphi^{\prime} \cdot \tilde{\varphi}_{r}=0$. Since $m_{r} \leqslant p-1$,

is a factor of

$$
\prod_{i=1}^{m_{r}} T^{i-1}\left[\left(I+\delta_{i}\right)\left(I+\delta_{i+r}\right)\right]
$$

$$
\varphi^{\prime} \cdot \tilde{\varphi}_{r}=\prod_{i=1}^{p-1} T^{i-1}\left(I+\delta_{i}\right) \prod_{i=1}^{m_{r}} T^{i-1}\left(I+\delta_{i+r}\right) .
$$

Suppose $\delta_{i}=\delta_{i+r}$ for $1 \leqslant i \leqslant m_{r}$. Then $r$ is a period of $\delta_{1} \cdots \delta_{m_{i}}$. If $m_{r}=p-1$, then by (6.2), $r$ is a period of $\delta_{1} \cdots \delta_{k}$, contradicting the minimality of $p$. If $m_{r}=k-r$, then $r$ is a period of $\delta_{1} \cdots \delta_{k}$, again contradicting the minimality of $p$.

Therefore $\delta_{i+r}=1+\delta_{i}$ for some $i, 1 \leqslant i \leqslant m_{r}$, and so $\left(I+\delta_{i}\right)\left(I+\delta_{i+r}\right)$ $=0$. Thus

$$
\prod_{i=1}^{m_{r}} T^{i-1}\left[\left(I+\delta_{i}\right)\left(I+\delta_{i+r}\right)\right]=0 .
$$

Therefore $\varphi^{\prime} \cdot \tilde{\varphi}_{r}=0$.

(6.6) LeMMA. Let $\alpha_{i}=T^{i-1}\left(I+\delta_{i}\right)$ for $1 \leqslant i \leqslant k$ and let $\left\{\beta_{j} \mid j \geqslant 1\right\}$ be a collection of block maps. If

(1) $\beta_{j} \beta_{j+r}=0$ for $j \geqslant 1$ and $1 \leqslant r \leqslant p-1$,

(2) $T^{r} \varphi^{\prime}$ is a factor of $\beta_{r}$ for $1 \leqslant r \leqslant p-1$, then

$$
\prod_{i=1}^{k}\left(\alpha_{i}+\beta_{i}\right)=\varphi^{\prime} \cdot \prod_{i=p}^{k}\left(\alpha_{i}+\beta_{i}\right) \text {. }
$$

If, furthermore, $p>\frac{1}{2} k$, then

$$
\prod_{i=1}^{k}\left(\alpha_{i}+\beta_{i}\right)=\varphi_{1}+\sum_{j=p}^{k} \beta_{j} \hat{\varphi}_{j}
$$


Proof. It suffices to show that condition (2) of (6.3) holds. Since for $1 \leqslant r \leqslant p-1$,

$$
\prod_{i=1}^{m_{r}} \alpha_{i+r}=\prod_{i=1}^{m_{r}} T^{i+r-1}\left(I+\delta_{i+r}\right)=T^{r} \prod_{i=1}^{m_{r}} T^{i-1}\left(I+\delta_{i+r}\right)=T^{r} \tilde{\varphi}_{r},
$$

it follows from hypothesis (2) and (6.5) that $\beta_{r} \Pi_{i=1}^{m} \alpha_{i+r}=0$ for $1 \leqslant r \leqslant p-$ 1.

(6.7) Proposition. Let $n \geqslant 1$ and $1 \leqslant r \leqslant p-1$. Then

$\left(\mathrm{a}_{n}\right) \varphi_{n} \cdot T^{r} \varphi_{1}=0$.

$\left(\mathrm{b}_{n}\right) \varphi_{1} \cdot T^{r} \varphi_{n}=0$.

(c $\left.c_{n}\right) \varphi_{n} \cdot T^{r} \varphi_{n}=0$.

$\left(\mathrm{d}_{n}\right) \varphi^{\prime}$ is a factor of $\varphi_{n}$.

$\left(\mathrm{e}_{n}\right) \varphi^{\prime}$ is a factor of $\varphi_{1} \circ f^{n}$.

PROof. The proof is by induction on $n$.

$\left(a_{1}\right)-\left(c_{1}\right)$ : Since $T^{r} \varphi^{\prime}$ is a factor of $T^{r} \varphi_{1}$ and $T^{r} \tilde{\varphi}_{r}$ is a factor of $\varphi_{1}$, $T^{r} \varphi_{1} \cdot T^{r} \tilde{\varphi}_{r}$ is a factor of $\varphi_{1} \cdot T^{r} \varphi_{1}$. Then by (6.5), $\varphi_{1} \cdot T^{r} \varphi_{1}=0$.

$\left(\mathrm{d}_{1}\right)$ : See (6.4).

$\left(\mathrm{e}_{1}\right)$ : For $1 \leqslant i \leqslant k$, let $\alpha_{i}=T^{i-1}\left(I+\delta_{i}\right)$ and for $j \geqslant 1$, let $\beta_{j}=T^{j} \varphi_{1}$. Then $\varphi_{1} \circ f=\prod_{i=1}^{k}\left(\alpha_{i}+\beta_{i}\right)$. It suffices to show that conditions (1) and (2) of (6.6) hold. By $\left(\mathrm{a}_{1}\right), \varphi_{1} \cdot T^{r} \varphi_{1}=0$ and so $T^{j} \varphi_{1} \cdot T^{j+r} \varphi_{1}=0$, i.e., $\beta_{j} \beta_{j+r}=0$ and (1) holds. Since $\varphi^{\prime}$ is a factor of $\varphi_{1}, T^{r} \varphi^{\prime}$ is a factor of $\beta_{r}$ and so (2) holds.

Suppose that $\left(\mathrm{a}_{n}\right)-\left(\mathrm{e}_{n}\right)$ are true. We show that $\left(\mathrm{a}_{n+1}\right)-\left(\mathrm{e}_{n+1}\right)$ hold.

$\left(a_{n+1}\right)$ : It follows from (2.8) and $\left(a_{n}\right)$ that

$$
\varphi_{n+1} \cdot T^{r} \varphi_{1}=\left(\varphi_{n}+\varphi_{1} \circ f^{n}\right) \cdot T^{r} \varphi_{1}=\left(\varphi_{1} \circ f^{n}\right) \cdot T^{r} \varphi_{1} .
$$

Since $T^{r} \varphi^{\prime}$ is a factor of $T^{r} \varphi_{1}$ and $T^{r} \varphi_{1} \cdot T^{r} \varphi_{1}=T^{r} \varphi_{1}$, by (6.5) it suffices to show that $T^{r} \tilde{\varphi}_{r}$ is a factor of $T^{r} \varphi_{1} \cdot\left(\varphi_{1} \circ f^{n}\right)$.

For $1 \leqslant i \leqslant k$, let $\alpha_{i}=T^{i-1}\left(I+\delta_{i}\right)$ and for $j \geqslant 1$, let $\beta_{j}=T^{j} \varphi_{n}$. Then $\varphi_{1} \circ f^{n}=\Pi_{i=1}^{k}\left(\alpha_{i}+\beta_{i}\right)$. By $\left(b_{n}\right), \varphi_{1} \cdot T^{j} \varphi_{n}=0$ for $1<j<p-1$, and so $T^{r} \varphi_{1} \cdot T^{j+r} \varphi_{n}=0$ for $1 \leqslant j \leqslant p-1$, or equivalently, $T^{r} \varphi_{1} \cdot T^{i} \varphi_{n}=0$ for $r+1 \leqslant i \leqslant p+r-1$. Thus $T^{r} \varphi_{1} \cdot \beta_{i}=0$ for $r+1 \leqslant i \leqslant p+r-1$. Since $m_{r}+r \leqslant p+r-1$,

$$
T^{r} \varphi_{1} \cdot \prod_{i=r+1}^{m_{r}+r}\left(\alpha_{i}+\beta_{i}\right)=T^{r} \varphi_{1} \cdot \prod_{i=r+1}^{m_{r}+r} \alpha_{i}=T^{r} \varphi_{1} \cdot T^{r} \tilde{\varphi}_{r} .
$$

Therefore $T^{r} \tilde{\varphi}_{r}$ is a factor of $T^{r} \varphi_{1} \cdot \prod_{i=r+1}^{m_{r}+r}\left(\alpha_{i}+\beta_{i}\right)$ which in turn is a factor of $T^{r} \varphi_{1} \cdot \Pi_{i=1}^{k}\left(\alpha_{i}+\beta_{i}\right)=T^{r} \varphi_{1} \cdot\left(\varphi_{1} \circ f^{n}\right)$. Thus $\left(\mathrm{a}_{n+1}\right)$ is true.

$\left(\mathrm{b}_{n+1}\right)$ : It follows from $(2.8)$ and $\left(\mathrm{b}_{n}\right)$ that

$$
\varphi_{1} \cdot T^{r} \varphi_{n+1}=\varphi_{1} \cdot\left(T^{r} \varphi_{n}+T^{r} \varphi_{1} \circ f^{n}\right)=\varphi_{1} \cdot\left(T^{r} \varphi_{1} \circ f^{n}\right)=\varphi_{1} \cdot T^{r}\left(\varphi_{1} \circ f^{n}\right)
$$

By $\left(\mathrm{e}_{n}\right), T^{r} \varphi^{\prime}$ is a factor of $T^{r}\left(\varphi_{1} \circ f^{n}\right)$. Since $T^{r} \tilde{\varphi}_{r}$ is a factor of $\varphi_{1}, T^{r} \varphi^{\prime} \cdot T^{r} \tilde{\varphi}_{r}$ is a factor of $\varphi_{1} \cdot T^{r} \varphi_{n+1}$ and so by (6.5), $\varphi_{1} \cdot T^{r} \varphi_{n+1}=0$. 
$\left(\mathrm{c}_{n+1}\right):$ By (2.8), $\varphi_{n} \circ f=\varphi_{1}+\varphi_{n+1}$, hence $T^{r} \varphi_{n} \circ f=T^{r} \varphi_{1}+T^{r} \varphi_{n+1}$. Therefore

$\left(\varphi_{n} \circ f\right) \cdot\left(T^{r} \varphi_{n} \circ f\right)=\varphi_{1} \cdot T^{r} \varphi_{1}+\varphi_{1} \cdot T^{r} \varphi_{n+1}+\varphi_{n+1} \cdot T^{r} \varphi_{1}+\varphi_{n+1} \cdot T^{r} \varphi_{n+1}$ and so

$\varphi_{n+1} \cdot T^{r} \varphi_{n+1}=\varphi_{1} \cdot T^{r} \varphi_{1}+\varphi_{1} \cdot T^{r} \varphi_{n+1}+\varphi_{n+1} \cdot T^{r} \varphi_{1}+\left(\varphi_{n} \circ f\right) \cdot\left(T^{r} \varphi_{n} \circ f\right)$.

The first three summands are zero by $\left(\mathrm{a}_{1}\right),\left(\mathrm{b}_{n+1}\right)$ and $\left(\mathrm{a}_{n+1}\right)$ respectively. But $\left(\varphi_{n} \circ f\right) \cdot\left(T^{r} \varphi_{n} \circ f\right)=\left(\varphi_{n} \cdot T^{r} \varphi_{n}\right) \circ f$ which is zero by $\left(\mathrm{c}_{n}\right)$. Therefore $\varphi_{n+1}$. $T^{r} \varphi_{n+1}=0$.

$\left(\mathrm{d}_{n+1}\right)$ : By (2.8), $\varphi_{n+1}=\varphi_{n}+\varphi_{1} \circ f^{n}$. By $\left(\mathrm{d}_{n}\right), \varphi^{\prime}$ is a factor of $\varphi_{n}$ and by $\left(\mathrm{e}_{n}\right), \varphi^{\prime}$ is a factor of $\varphi_{1} \circ f^{n}$. Therefore $\varphi^{\prime}$ is a factor of $\varphi_{n+1}$.

$\left(\mathrm{e}_{n+1}\right)$ : For $1 \leqslant i \leqslant k$, let $\alpha_{i}=T^{i-1}\left(I+\delta_{i}\right)$ and for $j \geqslant 1$, let $\beta_{j}=T^{j} \varphi_{n+1}$. Then $\varphi_{1} \circ f^{n+1}=\prod_{i=1}^{k}\left(\alpha_{i}+\beta_{i}\right)$. It suffices to show that conditions (1) and (2) of (6.6) hold. By $\left(c_{n+1}\right), \varphi_{n+1} \cdot T^{r} \varphi_{n+1}=0$ and so $T^{j} \varphi_{n+1} \cdot T^{j+r} \varphi_{n+1}=0$, i.e., $\beta_{j} \beta_{j+r}=0$ and so (1) holds. By $\left(\mathrm{d}_{n+1}\right), T^{r} \varphi^{\prime}$ is a factor of $T^{r} \varphi_{n+1}$, i.e., $T^{r} \varphi^{\prime}$ is a factor of $\beta_{r}$ and so (2) holds. Thus $\varphi^{\prime}$ is a factor of $\varphi_{n+1} \circ f$. 0 .

(6.8) Proposition. Let $1 \leqslant r \leqslant p-1$. Then for all $m, n \geqslant 1, \varphi_{m} \cdot T^{r} \varphi_{n}=$

Proof. By (6.7) the result is true if either $m=1$ or $n=1$. It therefore suffices to show that if $\varphi_{m} \cdot T^{r} \varphi_{n}=0$, then $\varphi_{m+1} \cdot T^{r} \varphi_{n+1}=0$.

As in the proof of $\left(c_{n+1}\right)$ of (6.7),

$$
\varphi_{m+1} \cdot T^{r} \varphi_{n+1}=\varphi_{1} \cdot T^{r} \varphi_{1}+\varphi_{1} \cdot T^{r} \varphi_{n+1}+\varphi_{m+1} \cdot T^{r} \varphi_{1}+\left(\varphi_{m} \cdot T^{r} \varphi_{n}\right) \circ f .
$$

By (6.7), the first three summands are zero. The fourth summand is zero by the inductive hypothesis. Therefore $\varphi_{m+1} \cdot T^{r} \varphi_{n+1}=0$.

(6.9) Proposition. If $p>\frac{1}{2} k$, then for all $n \geqslant 1$,

$$
\varphi_{1} \circ f^{n}=\varphi_{1}+\sum_{j=p}^{k} T^{j} \varphi_{n} \cdot \hat{\varphi}_{j} .
$$

Proof. For $1 \leqslant i \leqslant k$, let $\alpha_{i}=T^{i-1}\left(I+\delta_{i}\right)$ and for $j \geqslant 1$, let $\beta_{j}=T^{j} \varphi_{n}$. Then $\varphi_{1} \cdot f^{n}=\prod_{i=1}^{k}\left(\alpha_{i}+\beta_{i}\right)$ and it suffices to show that conditions (1) and (2) of (6.6) hold.

Let $1 \leqslant r \leqslant p-1$. By (6.7), $\varphi_{n} \cdot T^{r} \varphi_{n}=0$, so $T^{j} \varphi_{n} \cdot T^{j+r} \varphi_{n}=0$, i.e., $\beta_{j} \beta_{j+r}$ $=0$ and (1) holds. By (6.7) again, $T^{r} \varphi^{\prime}$ is a factor of $T^{r} \varphi_{n}$, i.e., $T^{r} \varphi^{\prime}$ is a factor of $\beta_{r}$ and (2) holds.

(6.10) Proposition. Let $s \geqslant 1$ and let $n_{i} \geqslant 1$ for $1 \leqslant i \leqslant 2 s$. If $p>\frac{1}{2} k$, then

$$
\varphi_{1} \circ\left(I+\sum_{i=1}^{2 s} f^{n_{i}}\right)=\varphi_{1}+\sum_{j=p}^{k} \sum_{i=1}^{2 s} T^{j} \varphi_{n_{i}} \cdot \hat{\varphi}_{j} \cdot
$$


Proof. For $1 \leqslant j \leqslant k$, let $\alpha_{j}=T^{j-1}\left(I+\delta_{j}\right)$ and for $j \geqslant 1$, let $\beta_{j}=$ $\sum_{i=1}^{2 s} T^{j} \varphi_{n_{i}}$. Since $\beta_{j}=T^{j-1}\left(\sum_{i=1}^{2 s} T \varphi_{n_{i}}\right)$ and

$$
\sum_{i=1}^{2 s} T \varphi_{n_{i}}=\sum_{i=1}^{2 s}\left(I+T \varphi_{n_{i}}\right)=\sum_{i=1}^{2 s} f^{n_{i}}
$$

it follows that

$$
\varphi_{1} \circ\left(I+\sum_{i=1}^{2 s} f^{n_{i}}\right)=\prod_{j=1}^{k}\left(\alpha_{j}+\beta_{j}\right) .
$$

Therefore it suffices to show that conditions (1) and (2) of (6.6) hold. Let $1 \leqslant r \leqslant p-1$. By (6.8), $\varphi_{m} \cdot T^{r} \varphi_{n}=0$ for all $m, n \geqslant 1$. Thus $T^{j} \varphi_{m} \cdot T^{j+r} \varphi_{n+1}$ $=0$ for all $j, m, n \geqslant 1$, and since

$$
\beta_{j} \beta_{j+r}=\left(\sum_{i=1}^{2 s} T^{j} \varphi_{n_{i}}\right) \cdot\left(\sum_{i=1}^{2 s} T^{j+r} \varphi_{n_{i}}\right),
$$

it follows that $\beta_{j} \beta_{j+r}=0$, i.e., (1) holds. By (6.7), $\varphi^{\prime}$ is a factor of $\varphi_{n}$ for all $n \geqslant 1$, so $T^{r} \varphi^{\prime}$ is a factor of $\sum_{i=1}^{2 s} T^{r} \varphi_{n_{i}}$, i.e., $T^{r} \varphi^{\prime}$ is a factor of $\beta_{r}$ and so (2) holds.

(6.11) TheOREM. Let $f\left(x_{0} \cdots x_{k}\right)=x_{0}+\mathrm{II}_{i=1}^{k}\left(x_{i}+\delta_{i}\right)$ where $k \geqslant 2$. Let $p$ be the least period of $\delta_{1} \cdots \delta_{k}$. If $p>\frac{1}{2} k$, then $\mathrm{C}^{*}(f)$ is the set of all sums of an odd number of powers of $f$.

Proof. Every sum of an odd number of powers of $f$ is linear in the first variable and hence has left extent one. So by (4.11), it suffices to show that every sum of an odd number of powers of $f$ commutes with $f$.

Let $g=\sum_{i=0}^{2 s} f^{n_{i}}$. If $s=0$, then $g=f^{n_{0}} \in \mathcal{C}(f)$, so we may assume $s \geqslant 1$. By (2.14), we may assume that $n_{0}=0$ and $n_{i} \geqslant 1$ for $1 \leqslant i \leqslant 2 s$. Then using (6.10) and (6.9),

$$
\begin{aligned}
\varphi_{1} \circ\left(I+\sum_{i=1}^{2 s} f^{n_{i}}\right) & =\varphi_{1}+\sum_{j=p}^{k} \sum_{i=1}^{2 s} T^{j} \varphi_{n_{i}} \cdot \hat{\varphi}_{j} \\
& =\varphi_{1}+\sum_{i=1}^{2 s} \sum_{j=p}^{k} T^{j} \varphi_{n_{i}} \cdot \hat{\varphi}_{j} \\
& =\varphi_{1}+\sum_{i=1}^{2 s}\left(\varphi_{1}+\sum_{j=p}^{k} T^{j} \varphi_{n_{i}} \cdot \hat{\varphi}_{j}\right)=\varphi_{1}+\sum_{i=1}^{2 s} \varphi_{1} \circ f^{n_{i}}
\end{aligned}
$$

Therefore

$$
T \varphi_{1}+\sum_{i=1}^{2 s} T \varphi_{1} \circ f^{n_{i}}+T \varphi_{1} \circ\left(I+\sum_{i=1}^{2 s} f^{n_{i}}\right)=0 .
$$


Then

$$
\begin{aligned}
g \circ f+f \circ g & =\left(I+\sum_{i=1}^{2 s} f^{n_{i}}\right) \circ f+\left(I+T \varphi_{1}\right) \circ\left(I+\sum_{i=1}^{2 s} f^{n_{i}}\right) \\
& =I+T \varphi_{1}+\sum_{i=1}^{2 s}\left(I+T \varphi_{1}\right) \circ f^{n_{i}}+I+\sum_{i=1}^{2 s} f^{n_{i}}+T \varphi_{1} \circ \sum_{i=1}^{2 s} f^{n_{i}} \\
& =T \varphi_{1}+\sum_{i=1}^{2 s} T \varphi_{1} \circ f^{n_{i}}+T \varphi_{1} \circ \sum_{i=1}^{2 s} f^{n_{i}}=0 .
\end{aligned}
$$

Therefore $g \in \mathcal{C}(f)$.

It is easy to see that for the block maps considered in this section, $\mathcal{C}^{\prime}(f)$ consists of both constant maps 0 and 1 .

\section{REFERENCES}

[F] J. D. Ferguson, Some properties of mappings on sequence spaces, $\mathrm{Ph}$. D. Dissertation, Yale University, 1962.

[H] G. A. Hedlund, Endomorphisms and automorphisms of the shift dynamical system, Math. Systems Theory 3 (1969), 320-375. MR 41 \#4510.

Department of Mathematics, Wesleyan University, Middletown, Connecticut 06457 (Current address of E. M. Coven and G. A. Hedlund)

Department of Mathematics, University of Southampton, SOUthampton S09 5NH, ENGLAND (Current address of Frank Rhodes) 